ISAHP 2001, Berne, Switzerland, August 2-4, 2001

\title{
IDENTIFYING AND RATING NON-SAMPLING ERRORS USING THE ANALYTIC HIERARCHY PROCESS
}

\author{
Cynthia Lynne Knott \\ George Washington University \\ School of Business and Public Management, Management Science Department \\ The George Washington University; Washington, DC 20052 - USA \\ cynth@gwu.edu
}

\begin{abstract}
When users discuss error for any type of sample-based data, they are generally referring to sampling error. Because of this many assume that sampling error is the most significant error and accounts for most of the total error. This is generally wrong but few efforts have been made to correct this misconception. When statisticians refer to error they are generally careful to specify they are referring to sampling error. And when they refer to total error they are generally referring to non-sampling error as well as sampling error. However, non-sampling error is generally not discussed beyond an acknowledgement that it exists. Even though many researchers argue that the non-sampling error is as large or larger than sampling error.

Sampling error is easily and routinely calculated using traditional statistical methods, i.e. standard error and variance using sample size and the structure of the sample. However, non-sampling error which includes errors such as; whether the correct respondents were surveyed, whether the respondent understood and interpreted the survey questions correctly, whether the data were processed properly, whether respondents answered truthfully, etc. are harder to define and much, much harder to quantify.

Given this omission, it is important to develop an approach to identifying non-sampling errors so these errors can be rated to determine their level of importance. If a specific error is a threat to the results of the survey, then action should be taken to ensure the error does not occur or its impact on the data is minimized. Using the Analytic Hierarchy Process Methodology (AHP), the various non-sampling errors can be identified during a brainstorming session. This would be done with the experts doing the research to determine the inventory of possible non-sampling errors. Once this is done, the relative importance of each of the errors or rating can be determined using the pair-wise comparison method in AHP. Consistency of the judgments by the participating experts can be examined using the eigen-value calculation in AHP and sensitivity analysis can also be performed to determine which errors are truly threats to the research.

The paper will have five sections: introduction, discussion and review of the materials on non-sampling error, presentation of the specific non-sampling errors from a brainstorming secession for a specific survey [final permission for use of a specific survey is pending], development and presentation of an AHP rating model and conclusions.
\end{abstract}


